\title{
Effective charges and zeta potentials of oil in water microemulsions in the presence of Hofmeister salts
}

Cite as: J. Chem. Phys. 148, 222817 (2018); https://doi.org/10.1063/1.5019704

Submitted: 15 December 2017 . Accepted: 26 February 2018 . Published Online: 16 March 2018

Alexandre P. dos Santos (D), and Yan Levin (D)

\section{ARTICLES YOU MAY BE INTERESTED IN}

Probing the Hofmeister series beyond water: Specific-ion effects in non-aqueous solvents The Journal of Chemical Physics 148, 222805 (2018); https://doi.org/10.1063/1.5017278

The influence of polarizability and charge transfer on specific ion effects in the dynamics of aqueous salt solutions

The Journal of Chemical Physics 148, 222803 (2018); https://doi.org/10.1063/1.5012682

Interfacial ion solvation: Obtaining the thermodynamic limit from molecular simulations

The Journal of Chemical Physics 148, 222823 (2018); https://doi.org/10.1063/1.5020563

Where in the world is AIP Publishing?

Find out where we are exhibiting next 


\title{
Effective charges and zeta potentials of oil in water microemulsions in the presence of Hofmeister salts
}

\author{
Alexandre P. dos Santos ${ }^{2, a)}$ and Yan Levin ${ }^{1, b)}$ \\ ${ }^{1}$ Instituto de Física, Universidade Federal do Rio Grande do Sul, Caixa Postal 15051, \\ CEP 91501-970 Porto Alegre, RS, Brazil \\ ${ }^{2}$ Fachbereich Physik, Freie Universität Berlin, 14195 Berlin, Germany
}

(Received 15 December 2017; accepted 26 February 2018; published online 16 March 2018)

\begin{abstract}
We present a theory which allows us to calculate the effective charge and zeta potential of oil droplets in microemulsions containing Hofmeister salts. A modified Poisson-Boltzmann equation is used to account for the surface and ion polarizations and hydrophobic and dispersion interactions. The ions are classified as kosmotropes and chaotropes according to their Jones-Dole viscosity B coefficient. Kosmotropes stay hydrated and do not enter into the oil phase, while chaotropes can adsorb to the oil-water interface. The effective interaction potentials between ions and oil-water interface are parametrized so as to accurately account for the excess interfacial tension. Published by AIP Publishing. https://doi.org/10.1063/1.5019704
\end{abstract}

\section{INTRODUCTION}

The interfacial tension of water-oil interface in the presence of different salts follows the Hofmeister series, ${ }^{1,2}$ first observed in the context of protein solubility. ${ }^{3}$ The same ionic series was found for micellization, ${ }^{4,5}$ colloidal suspensions, ${ }^{6-8}$ and bacterial growth. ${ }^{9}$ The series were investigated by theoretical, simulational, and experimental methods. ${ }^{10-18}$ Ionic specificity was first suggested to be due to dispersion interactions between ions and water; ${ }^{10}$ this, however, was later shown not to be the case. ${ }^{19-26}$ Recently we developed a theory which allows us to calculate the excess surface tensions of electrolyte-air interface in very good agreement with the experimental data. ${ }^{24,26-28}$ In theory, the chaotropic anions are allowed to adsorb to the interface, while kosmotropic ions remain hydrated in the aqueous medium. The adsorption can be explained as a competition between electrostatic and hydrophobic interactions. ${ }^{23,24,29}$ The theory was extended to electrolyte-oil interface with inclusion of dispersion forces. $^{2}$

Oil in water microemulsions (OWEs) are present in various biological, chemical, and industrial materials. Many of the culinary techniques rely on OWEs. Stability of OWEs depends on the environment and on the emulsion composition. Often charged surfactants are used to stabilize microemulsions to avoid coagulation. OWEs with positively charged oil droplets can be prepared using cationic surfactants. ${ }^{30-32}$ Charged microemulsions have been considered for skin therapy because of their more effective penetration. ${ }^{33}$ They have been used in asphalts ${ }^{34}$ and drug delivery. ${ }^{35}$ Recently, DNA-coated oil droplets have been a subject of intense research. ${ }^{36,37}$ Charged emulsions are strongly influenced by the released counterions and the type of added salt. In the

a)Electronic mail: alexandre.pereira@ufrgs.br

b)Electronic mail: levin@if.ufrgs.br present paper, we will combine the theory developed to calculate interfacial tension of electrolyte-oil interface with a modified Poisson-Boltzmann (PB) equation to obtain the effective charge and zeta potential of nanodroplets composed of oil and cationic surfactants. In Sec. II, we will present our model and the theory, followed by Secs. III and IV describing the results and conclusions.

\section{MODEL AND THEORY}

We are interested to calculate the electrostatic potential and the effective charge of nano-droplets of oil stabilized by cationic surfactants. To account for finite concentration of oil, we will use a Wigner-Seitz (WS) cell, the radius of which is adjusted to yield the appropriate volume fraction of oil in the emulsion, see Fig. 1. A spherical oil droplet of radius $a=100 \AA$ and charge $Z q$ (produced by a cationic surfactant layer) is located at the center of a spherical WS cell of radius $R$, where $q$ is the elementary charge. The volume fraction of oil is $\phi_{c}=a^{3} / R^{3} . Z$ counterions from the dissociation of cationic surfactant and $N_{s}$ counterions and $N_{s}$ coions from dissociation of salt are also present inside the WS cell. We will treat all the counterions as the same specie independent of whether they are derived from surfactant or salt dissociation. The salt concentration is

$$
\rho_{S}=\frac{3}{4 \pi} \frac{N_{S}}{\left(R^{3}-a^{3}\right)} .
$$

The effective ionic radii of positive and negative ions are $r_{+}$and $r_{-}$, respectively. The model used for ions is the same as in our earlier work, which allowed us to calculate surface and interfacial tensions of electrolyte solutions in a very good agreement with experiments. ${ }^{24,26-28}$ The chaotropic anions can loose their hydration sheath and adsorb to the oil-water interface so that the radii of chaotropic ions are very close to their crystallographic radii; on the other hand kosmotropic ions always remain hydrated so that their hydrated radii enter the theory. 


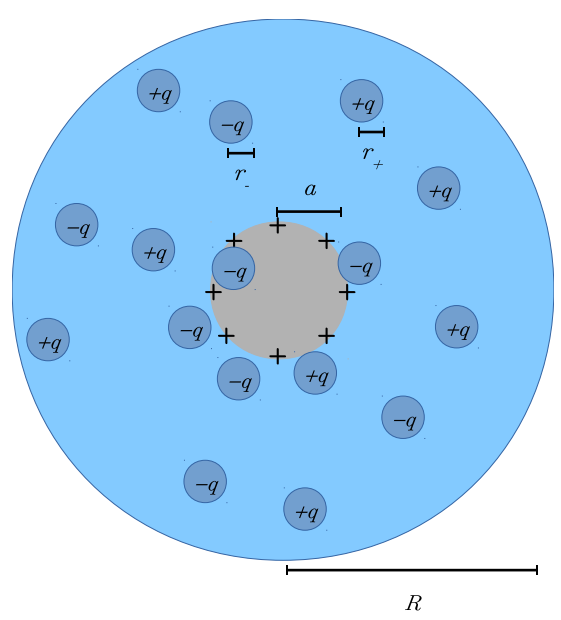

FIG. 1. Representation of the system. A charged spherical oil droplet surrounded by electrolyte.

In the present work, the coion is $\mathrm{Na}^{+}$with hydrated radius $r_{+}=2.5 \AA$. To solvate an ion in water requires creation of a cavity, formation of which perturbs the hydrogen bond network and costs hydrophobic free energy. If the ion partially escapes into the oil phase, the perturbation to the water structure diminishes. For strongly hydrated kosmotropic ions, the cavitational energy gain of moving an ion to the interface does not compensate the electrostatic self-energy cost of exposing ionic charge to the low dielectric oil environment. ${ }^{23}$ On the other hand, for large, highly polarizable chaotropic ions, the cavitational gain in hydrophobic free energy and the electrostatic self-energy penalty of moving a chaotropic anion to the interface become comparable. ${ }^{23}$

The electrostatic self-energy of a chaotropic anion of radius $r_{-}$and relative polarizability $\alpha$ at a distance $z$ from the oil-water interface — the Gibbs dividing surface (GDS)—was calculated to be ${ }^{23}$

$$
\begin{aligned}
\beta U_{p}(z)= & \frac{\lambda_{B}}{2 r_{-}}\left[\frac{\pi x^{2}}{\theta(z)}+\frac{\pi[1-x]^{2} \epsilon_{w}}{[\pi-\theta(z)] \epsilon_{o}}\right] \\
& +g\left[x-\frac{1-\cos [\theta(z)]}{2}\right]^{2}
\end{aligned}
$$

where $\theta(z)=\arccos \left[-z / r_{-}\right], g=(1-\alpha) / \alpha$, and $\lambda_{B}=q^{2} / \epsilon_{w} k_{B} T$ is the Bjerrum length set to $7.2 \AA$, value for water at room temperature. The dielectric constants for water and oil are $\epsilon_{w}$ and $\epsilon_{o}$, respectively, and $x$ is the fraction of ionic charge that remains hydrated. As the ion penetrates into the oil phase, its electronic charge becomes polarized, shifting to remain hydrated. The fraction of ionic charge that remains hydrated can be obtained by minimizing Eq. (2), and we find

$$
x(z)=\frac{\left[\frac{\lambda_{B} \pi \epsilon_{w}}{r_{-} \epsilon_{o}[\pi-\theta(z)]}+g[1-\cos [\theta(z)]]\right]}{\left[\frac{\lambda_{B} \pi}{r_{-} \theta(z)}+\frac{\lambda_{B} \pi \epsilon_{w}}{r_{-} \epsilon_{o}[\pi-\theta(z)]}+2 g\right]} .
$$

The cavitational energy gain of moving an ion to the interface is proportional to the volume exposed to oil, ${ }^{23,38}$

$$
U_{c}(z)=\left\{\begin{array}{l}
v r_{-}^{3} \text { for } z \geq r_{-}, \\
\frac{1}{4} v r_{-}^{3}\left(\frac{z}{r_{-}}+1\right)^{2}\left(2-\frac{z}{r_{-}}\right) \text {for }-r_{-}<z<r_{-},
\end{array}\right.
$$

where $v \approx 0.3 k_{B} T / \AA^{3}$ is obtained from bulk simulations. ${ }^{39}$ When ion penetrates into oil, there is also a lipophobic cavitational energy penalty which, however, is small and can be ignored. ${ }^{2}$ The repulsive potential which models the interface polarization was calculated to $\mathrm{be}^{24,27}$

$$
\beta U_{i m}(z)=\left\{\begin{array}{l}
\beta W_{c} \frac{r_{ \pm}}{z} e^{-2 \kappa\left(z-r_{ \pm}\right)} \text {for } z \geq r_{ \pm}, \\
\beta W_{c} \frac{z}{r_{-}} \text {for } 0<z<r_{-}, \\
0 \text { for }-r_{-}<z \leq 0
\end{array}\right.
$$

where $\kappa=\sqrt{8 \pi \lambda_{B} \rho_{s}}$ is the inverse Debye length and $W_{c}$ is the work ${ }^{40}$ needed to bring an ion from infinity to the contact distance $z=r_{ \pm}$,

$$
\beta W_{c}=\frac{\lambda_{B}}{2} \int_{0}^{\infty} d k \frac{k\left[s \cosh \left(k r_{ \pm}\right)-k \sinh \left(k r_{ \pm}\right)\right]}{s\left[s \cosh \left(k r_{ \pm}\right)+k \sinh \left(k r_{ \pm}\right)\right]},
$$

where $s=\sqrt{\kappa^{2}+k^{2}}$. Note that the first equation of expression (5) is valid for both chaotropic and kosmotropic ions; however, while chaotropes can cross GDS, the kosmotropes-because of their strong hydration-experience a hardcore-like repulsion from the interface and are restricted to distances $z$ larger than their hydrated radius. ${ }^{24,27}$

For a water-oil interface, the dispersion interaction between ions, water, and oil must also be taken into account for chaotropic ions. The dispersion interaction potential was calculated to be ${ }^{2}$

$U_{d}(z)=\left\{\begin{array}{l}0 \text { for } z \geq r_{-}, \\ A_{e} \alpha\left[1-\frac{\left(z / r_{-}+1\right)^{2}\left(2-z / r_{-}\right)}{4}\right] \text { for }-r_{-}<z<r_{-},\end{array}\right.$

where $A_{e}=4 k_{B} T$ is the effective Hamaker constant.

The ionic density profiles outside a charged oil droplet can be calculated using the modified Poisson-Boltzmann (PB) equation in spherical coordinates,

$$
\begin{aligned}
\nabla^{2} \phi(r) & =-\frac{4 \pi q}{\epsilon_{w}}\left[\frac{Z}{4 \pi a^{2}} \delta(r-a)+\rho_{+}(r)-\rho_{-}(r)\right], \\
\rho_{+}(r) & =\frac{N_{s} e^{\left[-\beta q \phi(r)-\beta U_{i m}(z)\right]}}{4 \pi \int_{a+r_{+}}^{R} d r r^{2} e^{\left[-\beta q \phi(r)-\beta U_{i m}(z)\right]}}, \\
\rho_{-}^{k}(r) & =\frac{\left(N_{s}+Z\right) e^{\left[\beta q \phi(r)-\beta U_{i m}(z)\right]}}{4 \pi \int_{a+r_{-}}^{R} d r r^{2} e^{\left[\beta q \phi(r)-\beta U_{i m}(z)\right]}}, \\
\rho_{-}^{c}(r) & =\frac{\left(N_{s}+Z\right) e^{\left[\beta q \phi(r)-\beta U_{i m}(z)-\beta U_{p}(z)-\beta U_{c}(z)-\beta U_{d}(z)\right]}}{4 \pi \int_{a-r_{-}}^{R} d r r^{2} e^{\left[\beta q \phi(r)-\beta U_{i m}(z)-\beta U_{p}(z)-\beta U_{c}(z)-\beta U_{d}(z)\right]}},
\end{aligned}
$$

where $\phi(r)$ is the electrostatic potential and $z=r-a$. The superscripts $k$ and $c$ denote kosmotropic and chaotropic anions, respectively. In Table I, we summarize the values of the ionic radii, polarizabilities, and ionic classification for ions studied in the present paper.

The effective droplet charge is defined as the charge for which the analytical solution of the linearized PB equation matches exactly the asymptotic solution of the full non-linear PB equation, Eq. (8). It can be calculated in terms of ionic concentration at the WS cell boundary. ${ }^{42-44}$ We find 
TABLE I. Ion classification into chaotropes (c) and kosmotropes (k). Effective radii (hydrated or partially hydrated) for kosmotropes and (bare) for chaotropes, for which we have also included the polarizabilities. ${ }^{41}$

\begin{tabular}{lccc}
\hline \hline Anions & Chao/kosmo & Radius $(\AA)$ & Polarizability $\left(\AA^{3}\right)$ \\
\hline $\mathrm{F}^{-}$ & $\mathrm{k}$ & 3.52 & $\ldots$ \\
$\mathrm{Cl}^{-}$ & $\mathrm{k}$ & 2 & $\ldots$ \\
$\mathrm{Br}^{-}$ & $\mathrm{c}$ & 2.05 & 5.07 \\
$\mathrm{I}^{-}$ & $\mathrm{c}$ & 2.26 & 7.4 \\
\hline \hline
\end{tabular}

$$
\begin{aligned}
Z_{e f f}= & \frac{G}{2 \kappa_{p}^{3}}\left[\left(\kappa_{p} R+1\right)\left(\kappa_{p} a-1\right) e^{-\kappa_{p}(R-a)}\right. \\
& \left.-\left(\kappa_{p} R-1\right)\left(\kappa_{p} a+1\right) e^{\kappa_{p}(R-a)}\right]
\end{aligned}
$$

where

$$
\begin{gathered}
G=-\frac{4 \pi \lambda_{B} \beta}{q}\left(\rho_{+}^{B}-\rho_{-}^{B}\right), \\
\kappa_{p}=\sqrt{4 \pi \lambda_{B}\left(\rho_{+}^{B}+\rho_{-}^{B}\right)},
\end{gathered}
$$

and $\rho_{ \pm}^{B}$ are the ionic concentrations at the WS cell boundary. For separations larger than Debye length, the oil droplets will interact with each through the Derjaguin-Landau-VerweyOverbeek (DLVO) potential with the bare droplet charge replaced by $Z_{\text {eff }}$.

\section{RESULTS}

We start by calculating the effective droplet charge as a function of bare charge $Z$ for four halide salts at various concentrations. The results are presented in Fig. 2. The effective droplet charge follows the Hofmeister series. For sufficiently weakly charged droplets and salts with chaotropic anions $(\mathrm{NaBr}, \mathrm{NaI})$, the effective charge is reversed-the droplet becomes negative charged-reflecting a strong adsorption of chaotropic anions to the oil-water interface. We observe that effective charge saturates for large bare charge $Z$ for all salts. In Fig. 3, we present the saturated values of the effective
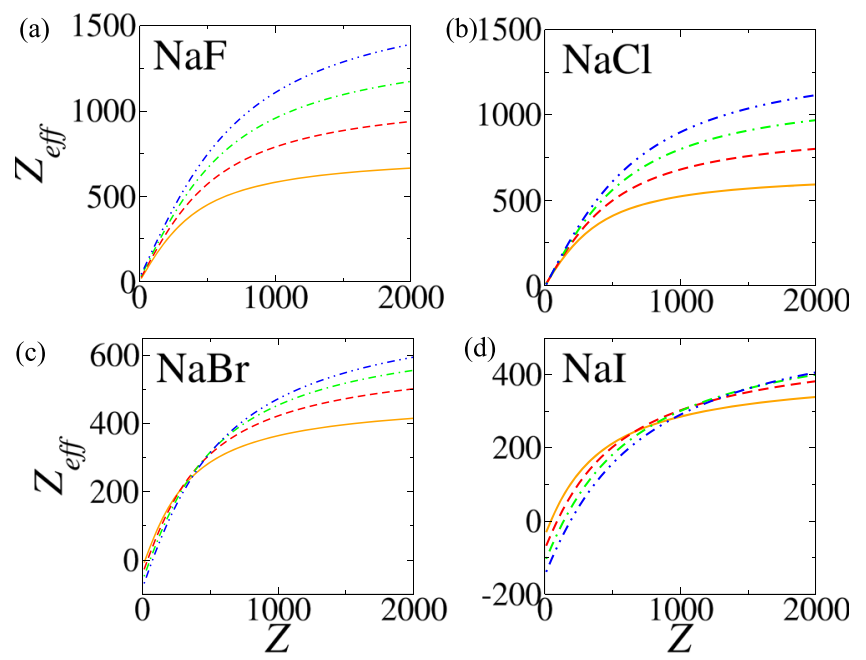

FIG. 2. Effective charges as a function of droplet bare charge $Z$. The oil volume fraction is $\phi_{c}=0.125$. Solid, dashed, dotted-dashed, and dotteddotted-dashed lines are for salt concentrations $\rho_{s}=50,100,150$, and $200 \mathrm{mM}$, respectively.

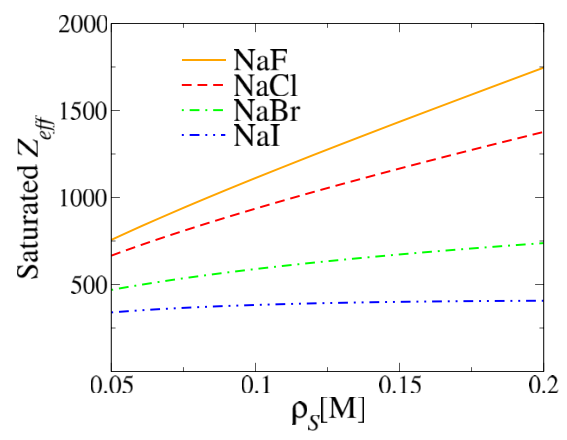

FIG. 3. Saturated effective charges $(Z \rightarrow \infty)$ as a function of added salt $\rho_{s}$. The oil volume fraction is $\phi_{c}=0.125$. Solid, dashed, dotted-dashed, and dotteddotted-dashed lines represent salts, $\mathrm{NaF}, \mathrm{NaCl}, \mathrm{NaBr}$, and $\mathrm{NaI}$, respectively.

charge for halide salts as a function of added salt concentration. In Fig. 4, we show the effective charge of droplets as a function of added salt. For kosmotropic salts, the effective charge increases as a function of added salt, in the range of parameters considered in this study. This is a consequence of screening of Coulomb interaction which diminishes the counterion condensation that is responsible for charge renormalization. For salts with chaotropic anions, the behavior of the effective charge is more complex. For weakly charged droplets, the effective charge decreases with increasing salt concentration, while for highly charged droplets it increases, reaches a maximum, and then starts to decrease; see bottom panels in Fig. 4. In this case, there is a strong competition between anion adsorption, produced by the hydrophobic and dispersion interactions, and the Debye screening which diminishes Coulomb attraction between counterions and cationic surfactants at the droplet surface. For iodide, we find that hydrophobic and dispersion interactions are so strong as to actually reverse the droplet charge at sufficiently large salt concentrations.

We next study the electrostatic surface potential which we will associate with the zeta potential measured in
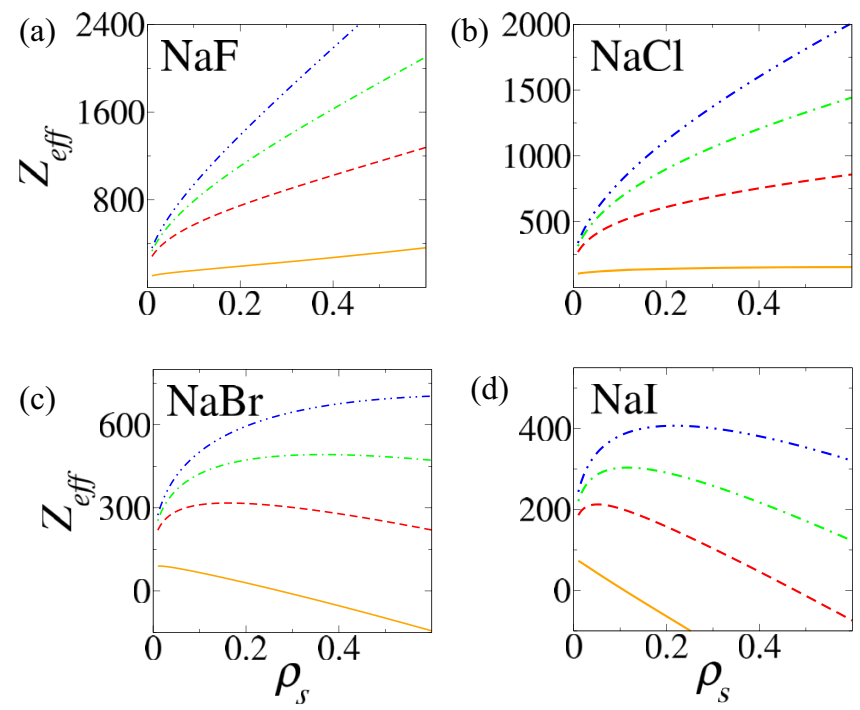

FIG. 4. Effective charge as a function of added salt concentration $\rho_{s}$. The emulsion volume fraction is $\phi_{c}=0.125$. Solid, dashed, dotted-dashed, and dotted-dotted-dashed lines represent droplet bare charge $Z=100,500,1000$, and 2000 , respectively. 


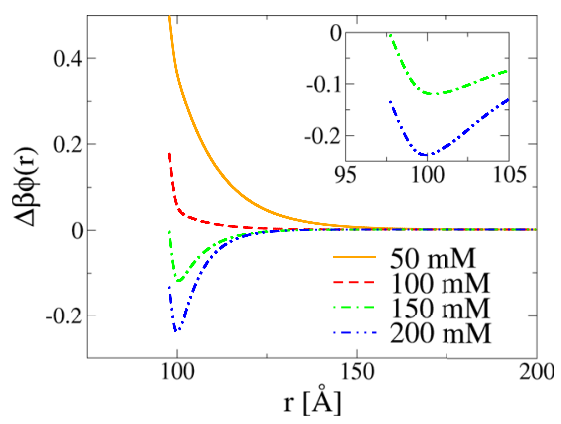

FIG. 5. Electrostatic potential difference for droplets with bare charge $Z=100$ and four different added NaI salt concentrations. The inset is an amplification. Solid, dashed, dotted-dashed, and dotted-dotted-dashed lines represent $\rho_{S}=50,100,150$, and $200 \mathrm{mM}$, respectively.

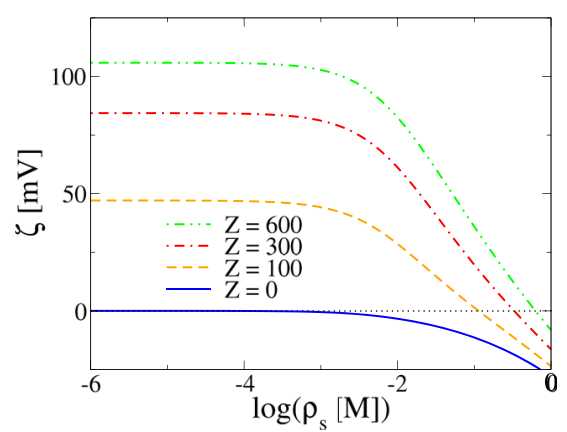

FIG. 6. Zeta potential for NaI. Solid, dashed, dotted-dashed, and dotteddotted-dashed lines represent $Z=0,100,300$, and 600, respectively. The volume fraction is $\phi_{c}=0.125$.

electrophoretic mobility experiments. The electrostatic potential difference

$$
\Delta \phi(r)=\phi(r)-\phi(R),
$$

inside a microemulsion containing $\mathrm{NaI}$, as a function of distance from the center of the droplet is shown in Fig. 5. For large salt concentrations, $\Delta \phi$ changes sign and exhibits a minimum at the GDS. We will, therefore, associate ${ }^{45}$ the electrockinetic zeta potential with $\zeta=\Delta \phi(a)$. Zeta potentials for various bare charges are shown in Fig. 6, as a function of added NaI concentration. Unfortunately experimental data for zeta potentials of charged oil droplets is very rare, available only for very low salt concentrations and does not explore the ionic specificity. ${ }^{46-48}$ We predict that for sufficiently large chaotropic salt concentrations, zeta potential of cationic oil droplets will reverse sign,

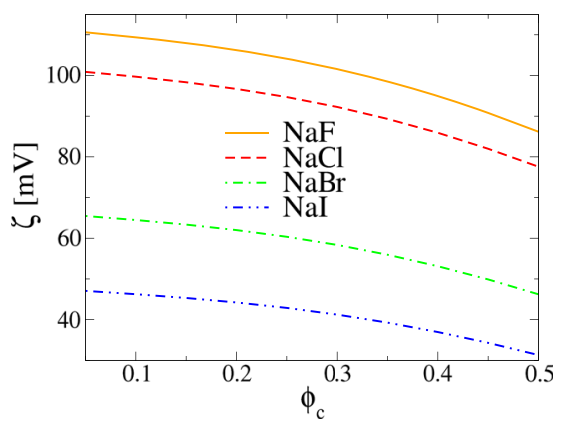

FIG. 7. Zeta potential for $\mathrm{NaF}, \mathrm{NaCl}, \mathrm{NaBr}$, and $\mathrm{NaI}$ represented by solid, dashed, dotted-dashed, and dotted-dotted-dashed lines, respectively. The droplet charge is $Z=500$ and the concentration of added salt is $50 \mathrm{mM}$. resulting in a reversal of electrophoretic mobility. Finally, in Fig. 7, we show that zeta potential decreases when oil volume fraction in the microemulsion increases.

\section{CONCLUSIONS}

We have studied the effective charge and zeta potential of oil in water microemulsions stabilized by cationic surfactants. The interaction of ions with the oil-water interface was treated using previously derived potentials which quantitatively account for Coulomb, dispersion, and hydrophobic interactions. We find that for a given salt concentration, the effective charge saturates as a function of bare droplet charge. For Hofmeister salts with chaotropic anions, the effective charge presents a maximum and then decrease with increasing salt concentration. This shows the importance of hydrophobic and dispersion interactions which drive chaotropic anions towards the oil-water interface. This behavior is very different from what is found for salts with kosmotropic anions for which there is a monotonic increase of the effective charge as more salt is added to emulsion. In this case, the augmentation of the effective charge is produced by the increase of the Debye screening, which diminishes the Coulomb attraction between oil droplets and kosmotropic counterions, lowering counterion condensation at higher salt concentrations. We have also calculated zeta potentials of oil droplets in microemulsions with Hofmeister salts. We find that the electrophoretic mobility of weakly charged cationic oil droplets can be reversed by addition of salts with chaotropic anions. Finally, we note that that $\mathrm{pH}$ is a very important parameter for stability of charged emulsions. In our calculations, the $\mathrm{pH}$ was kept fixed so that the bare charge includes binding of $\mathrm{H}^{+}$and $\mathrm{OH}^{-}$. In future work, we will extend the calculations to account for variability of $\mathrm{pH}$. This, in principle, should be feasible using the recently developed model of hydronium adsorption, which has proven quite accurate for predicting surface tensions of acid solutions. ${ }^{26,49}$

\section{ACKNOWLEDGMENTS}

This work was partially supported by the CNPq, CAPES, Alexander von Humboldt Foundation, INCT-FCx, and by the US-AFOSR under the Grant No. FA9550-16-1-0280.

${ }^{1}$ R. Aveyard and S. M. Saleem, J. Chem. Soc., Faraday Trans. 1 72, 1609 (1976).

${ }^{2}$ A. P. dos Santos and Y. Levin, Langmuir 28, 1304 (2012).

${ }^{3}$ F. Hofmeister, Arch. Exp. Pathol. Pharmakol. 24, 247 (1888).

${ }^{4}$ J. Luczak, M. Markiewicz, J. Thöming, J. Hupka, and C. Jungnickel, J. Colloid Interface Sci. 362, 415 (2011).

${ }^{5}$ A. P. dos Santos, W. Figueiredo, and Y. Levin, Langmuir 30, 4593 (2014).

${ }^{6}$ T. Lopez-Leon, A. B. Jodar-Reyes, D. Bastos-Gonzalez, and J. L. OrtegaVinuesa, J. Phys. Chem. B 107, 5696 (2003).

${ }^{7}$ T. Lopez-Leon, M. J. Santander-Ortega, J. L. Ortega-Vinuesa, and D. Bastos-Gonzalez, J. Phys. Chem. C 112, 16060 (2008).

${ }^{8}$ A. P. dos Santos and Y. Levin, Phys. Rev. Lett. 106, 167801 (2011).

${ }^{9}$ P. Lo Nostro, B. W. Ninham, A. L. G. Pesavento, L. Fratoni, and P. Baglioni, Phys. Biol. 2, 1 (2005).

${ }^{10}$ M. Boström, D. R. M. Williams, and B. W. Ninham, Langmuir 17, 4475 (2001).

${ }^{11}$ G. L. Richmond, Chem. Rev. 102, 2693 (2002).

${ }^{12}$ D. Liu, G. Ma, L. M. Levering, and H. C. Allen, J. Phys. Chem. B 108, 2252 (2004).

${ }^{13}$ E. A. Raymond and G. L. Richmond, J. Phys. Chem. B 108, 5051 (2004). 
${ }^{14}$ Y. R. Shen and V. Ostroverkhov, Chem. Rev. 106, 1155 (2006).

${ }^{15}$ S. Gopalakrishnan, D. Liu, H. C. Allen, M. Kuo, and M. J. Shultz, Chem. Rev. 106, 1155 (2006).

${ }^{16}$ P. Koelsch, P. Viswanath, H. Motschmann, V. L. Shapovalov, G. Brezesinski, H. Möhwald, D. Horinek, R. R. Netz, K. Giewekemeyer, T. Salditt, H. Schollmeyer, R. von Klitzing, J. Daillant, and P. Guenoun, Colloids Surf., A 303, 110 (2007).

${ }^{17}$ D. Horinek, A. Herz, L. Vrbka, F. Sedlmeier, S. I. Mamatkulov, and R. R. Netz, Chem. Phys. Lett. 479, 173 (2009).

${ }^{18}$ R. R. Netz and D. Horinek, Annu. Rev. Phys. Chem. 63, 401 (2012).

${ }^{19}$ P. Jungwirth and D. J. Tobias, J. Phys. Chem. B 105, 10468 (2001).

${ }^{20}$ P. Jungwirth and D. J. Tobias, J. Phys. Chem. B 106, 6361 (2002).

${ }^{21}$ L. X. Dang, J. Phys. Chem. B 106, 10388 (2002).

${ }^{22}$ L. X. Dang and T. M. Chang, J. Phys. Chem. B 106, 235 (2002).

${ }^{23}$ Y. Levin, Phys. Rev. Lett. 102, 147803 (2009).

${ }^{24}$ Y. Levin, A. P. dos Santos, and A. Diehl, Phys. Rev. Lett. 103, 257802 (2009).

${ }^{25}$ D. J. Tobias, A. C. Stern, M. D. Baer, Y. Levin, and C. J. Mundy, Annu. Rev. Phys. Chem. 64, 339 (2013).

${ }^{26}$ Y. Levin and A. P. dos Santos, J. Phys.: Condens. Matter 26, 203101 (2014).

${ }^{27}$ A. P. dos Santos, A. Diehl, and Y. Levin, Langmuir 26, 10778 (2010).

${ }^{28}$ A. P. dos Santos and Y. Levin, Faraday Discuss. 160, 75 (2013).

${ }^{29}$ M. D. Baer, A. C. Stern, Y. Levin, D. J. Tobias, and C. J. Mundy, J. Phys. Chem. Lett. 3, 1565 (2012).

${ }^{30}$ J. Surh, Y. S. Gu, E. A. Decker, and D. J. McClements, J. Agric. Food Chem. 53, 4236 (2005).

${ }^{31}$ L. Nilsson and B. Bergenstahl, J. Colloid Interface Sci. 308, 508-513 (2007).
${ }^{32}$ Z. Mei, S. Liu, L. Wang, J. Jiang, J. Xu, and D. Sun, J. Colloid Interface Sci. 361, 565 (2011).

${ }^{33}$ S. Da Costa, M. Basri, N. Shamsudin, and H. Basri, J. Chem. 2014, 748680.

${ }^{34}$ F. Wang, Y. Liu, Y. Zhang, and S. Hu, Constr. Build. Mater. 28, 117 (2012).

${ }^{35}$ P. R. Lockman, J. M. Koziara, R. J. Mumper, and D. D. Allen, J. Drug Targeting 12, 635 (2004).

${ }^{36}$ A. Levin, T. P. J. Knowles, C. Mugemana, N. Bruns, and W. J. Frith, e-print arXiv:1710.07820v1 (2017).

${ }^{37}$ Y. Zhang, A. McMullen, L. Pontani, X. He, R. Sha, N. C. Seeman, J. Brujic, and P. M. Chaikin, Nat. Commun. 8, 21 (2017).

${ }^{38}$ D. Chandler, Nature 437, 640 (2005).

${ }^{39}$ S. Rajamani, T. M. Truskett, and S. Garde, Proc. Natl. Acad. Sci. U. S. A. 102, 9475 (2005).

${ }^{40}$ Y. Levin and J. E. Flores-Mena, Europhys. Lett. 56, 187 (2001).

${ }^{41}$ N. C. Pyper, C. G. Pike, and P. P. Edwards, Mol. Phys. 76, 353 (1992).

${ }^{42}$ S. Alexander, P. M. Chaikin, P. Grant, G. J. Morales, P. Pincus, and D. Hone, J. Chem. Phys. 80, 5776 (1984).

${ }^{43}$ E. Trizac, L. Bocquet, M. Aubouy, and H. von Grunberg, Langmuir 19, 402 (2003).

${ }^{44}$ A. Diehl and Y. Levin, J. Phys.: Condens. Matter 17, S3309 (2005).

${ }^{45}$ A. Diehl and Y. Levin, J. Chem. Phys. 125, 054902 (2006).

${ }^{46}$ J. M. de Morais, O. D. H. dos Santos, T. Delicato, and P. A. da Rocha-Filho, J. Dispersion Sci. Technol. 27, 1009 (2006).

${ }^{47}$ D. Guzey and D. J. McClements, J. Agric. Food Chem. 55, 475 (2007).

${ }^{48} \mathrm{~K}$. Wojciechowski, A. Bitner, P. Warszyński, and M. Żubrowska, Colloid Surf., A 376, 122-126 (2011).

${ }^{49}$ A. P. dos Santos and Y. Levin, J. Chem. Phys. 133, 154107 (2010). 